\title{
Large-scale Samples Irradiation Facility at the IBR-2 Reactor in Dubna
}

\author{
A.P. Cheplakov, V.V. Golikov, S.M. Golubyh, G.Ya. Kaskanov, \\ E.N. Kulagin, V.V. Kukhtin, V.I. Luschikov, E.P. Shabalin \\ Joint Institute for Nuclear Research, Dubna, Russia \\ E. León-Florián, C. Leroy \\ University of Montreal, Montreal, Canada
}

May 18, 1998

\begin{abstract}
The irradiation facility at the beam line no. 3 of the IBR-2 reactor of the Frank Laboratory for Neutron Physics is described. The facility is aimed at irradiation studies of various objects with area up to $800 \mathrm{~cm}^{2}$ both at cryogenic and ambient temperatures. The energy spectra of neutrons are reconstructed by the method of threshold detector activation. The neutron fluence and $\gamma$ dose rates are measured by means of alanine and thermoluminescent dosimeters. The boron carbide and lead filters or $(n / \gamma)$ converter provide beams of different ratio of doses induced by neutrons and photons. For the lead filter, the flux of fast neutrons with energy more than $0.1 \mathrm{MeV}$ is $1.4 \cdot 10^{10} \mathrm{n} \mathrm{cm}^{-2} \mathrm{~s}^{-1}$ and the neutron dose is about $96 \%$ of the total radiation dose. For the $(n / \gamma)$ converter, the $\gamma$ dose rate is $\sim 500 \mathrm{~Gy} \mathrm{~h}^{-1}$ which is about $85 \%$ of the total dose. The radiation hardness tests of GaAs electronics and materials for the ATLAS detector to be put into operation at the Large Hadron Collider (LHC) have been performed successfully at this facility.
\end{abstract}




\section{Introduction}

Many components of the ATLAS detector will be located in the mixed high radiation field of photons and neutrons produced by the high luminosity running conditions at LHC. Examples are the materials used in the hadronic liquid argon end-cap calorimeter. At the high luminosity of $1.0 \cdot 10^{34} \mathrm{~cm}^{-2} \mathrm{~s}^{-1}$ a maximum year dose of $12 \mathrm{kGy} \mathrm{yr}^{-1}$ and a neutron fluence of $0.6 \cdot 10^{15} \mathrm{n} \mathrm{cm}^{-2} \mathrm{yr}^{-1}$ are expected in the hadronic endcaps [1]. The materials and electronics must withstand these radiation levels with a minimum deterioration of their performances.

The radiation hardness tests of GaAs preamplifiers developed in the framework of the RD33 Project [2] for the ATLAS liquid argon calorimetry were performed [3] at the pulsed fast neutron reactors IBR-2 and IBR-30 [4] of the Joint Institute for Nuclear Research at Dubna. The successful radiation tests of electronics and different types of glue [5] have demonstrated the advantage of using Dubna facilities for this purpose. Firstly, the energies of the fast neutrons are close to the energies of neutrons produced inside the hadronic showers in the ATLAS calorimeter. Secondly, high dose rates $\left(\sim 10^{10} \mathrm{n} \mathrm{cm}^{-2} \mathrm{~s}^{-1}\right.$ with neutron energy $\mathrm{E}_{n}>100 \mathrm{keV}$ ) are uniform over a large geometrical acceptance of the beam $\left(\sim 800 \mathrm{~cm}^{2}\right)$ and allow one to reach the dose level expected after 10 years of LHC operation within a few days.

The general parameters of the irradiation facilities at the Frank Laboratory for Neutron Physics of JINR at Dubna aimed at the radiation hardness study of various elements of the LHC detectors are described in this article. The facility available at the beam line no. 3 of the IBR-2 reactor is presented in more detail.

The boron carbide and lead filters or $(n / \gamma)$ converter provide the beams of different ratio of doses induced by neutrons and photons. The energy spectra of neutrons were reconstructed by using the method of the threshold detector activation. The neutron fluence and $\gamma$ dose rates were measured by means of alanine and thermoluminescent dosimeters.

\section{Description of the facility}

The IBR-2 reactor[4] is a pulsed source of fast neutrons with a very compact 22 litre active core. It is surrounded by two concrete rings of biological shield, each $2.5 \mathrm{~m}$ thick. The 3 $\mathrm{m}$ inner ring corridor between the shielding walls is intended for the location of various equipment. In particular, the facility purposed to study radiation hardness is developed in one of 14 available horizontal channels - at beam line no.3.

The setup for the radiation tests of the GaAs electronics is presented schematically in Fig. 1.

The beam line no. 3 has a rectangular apperture of $20 \times 40 \mathrm{~cm}^{2}$ in the inner shield of the reactor. The large-scale samples, such as a cryogenic cryostat, could be brought into the irradiation zone through the hole of $1 \mathrm{~m}$ diameter secured in the outer shielding 
wall. The cryostat with the samples to be irradiated is assembled on the movable platform inside a special shield box in the experimental hall. Also, a special beam shutter $1 \mathrm{~m}$ thick has been installed behind the platform to decrease the background in the experimental hall during the irradiation period. While assembling, the beam is blocked by the beam shutter of IBR-2 reactor in the inner shielding wall. The hole in the outer shielding wall is corked as well. When the beam shutter blocks the irradiation from the reactor, the platform with the cryostat is moving to the working position.

Different beam filters could be used according to the experimental requirements. For the irradiation by fast neutrons, a $5 \mathrm{~cm}$ thick layer of lead was installed in front of the cryostat to suppress the photon background. When the $\gamma$ effects were studied, this filter was replaced by the $(n / \gamma)$ converter $(12 \mathrm{~cm}$ thick) consisting of a paraffin moderator and a cadmium absorber for thermal neutrons. In addition, the cryostat was surrounded by $10 \mathrm{~cm}$ of paraffin and protected with a $5 \mathrm{~mm}$ thick layer of boron carbide $\left(\mathrm{B}_{4} \mathrm{C}\right)$ to reduce the activation of the equipment by thermal neutrons.

The cryostat installed on the support frame of the movable shielding platform was positioned for irradiation in the ring corridor $10 \mathrm{~cm}$ from the reactor beam shutter. During the experiment the cryostat was filled remotely with liquid nitrogen using special cryogenic lines. The motherboard with the electronic chips to be tested was kept inside the cryostat at liquid nitrogen temperature for the whole period of irradiation. Additional tubes in the platform were used for signal cables as well as for transportation of various material samples into the irradiation zone. Different characteristics of electronics, namely parameters of the preamplifiers like the transfer function, the rise time, the linearity and the equivalent noise current, were measured before and during the irradiation. For these short periods, the beam was blocked by the reactor beam shutter. The measurement cables had a total length of $40 \mathrm{~m}$. The data taking equipment was assembled in a counting room located about $15 \mathrm{~m}$ behind the outer shielding ring. The neutron and gamma dosimeters were mounted temporarily around the cryostat for the dose rate monitoring. A wide nickel foil covering the back side of the motherboard was kept in the cryostat during the whole run and served as a monitor of the total fast neutron flux via the activation reaction ${ }^{58} \mathrm{Ni}(\mathrm{n}, \mathrm{p}){ }^{58} \mathrm{Co}$. The induced activity of the nickel foil was used also to measure the homogeneity of the neutron fluence. It has been found that the neutron fluence is homogeneous within $\pm 5 \%$ over the area of $20 \times 10 \mathrm{~cm}^{2}$ (the size of the motherboard).

The facility allows one to perform simultaneous irradiation of other samples during the irradiation period of the large-scale samples. For this purpose a special container 13 $\mathrm{cm}$ in diameter and $80 \mathrm{~cm}$ of maximal length could be delivered to the position behind the cryostat through a special slightly curved tube inside the platform $6 \mathrm{~m}$ long. After the exposure, the container is kept inside the special shield box. The neutron and $\gamma$ activities of the reactor are significantly suppressed in the shielded box due to the tube curvature. 


\section{Fast neutron flux and energy spectrum measure- ments}

The standard method of the threshold detector activation was applied to measure the kinetic energy spectrum and the absolute value of the neutron fluence. The spectrum was reconstructed by applying an unfolding procedure to the yield measurements of the following reactions:

$$
\begin{gathered}
{ }^{115} \mathrm{In}\left(\mathrm{n}, \mathrm{n}^{\prime}\right){ }^{115 m} \mathrm{In},{ }^{58} \mathrm{Ni}(\mathrm{n}, \mathrm{p}){ }^{58} \mathrm{Co},{ }^{54} \mathrm{Fe}(\mathrm{n}, \mathrm{p}){ }^{54} \mathrm{Mn},{ }^{46} \mathrm{Ti}(\mathrm{n}, \mathrm{p}){ }^{46} \mathrm{Sc}, \\
{ }^{47} \mathrm{Ti}(\mathrm{n}, \mathrm{p}){ }^{47} \mathrm{Sc},{ }^{48} \mathrm{Ti}(\mathrm{n}, \mathrm{p}){ }^{48} \mathrm{Sc},{ }^{27} \mathrm{Al}(\mathrm{n}, \alpha){ }^{24} \mathrm{Na} .
\end{gathered}
$$

The threshold values for these reactions are between 0.4 and $6 \mathrm{MeV}$.

The yields were determined from the measurements of the induced $\gamma$ activity by means of a GeLi detector. The peak area $\mathrm{S}_{\gamma}$ of decay photons with energy $\mathrm{E}_{\gamma}$ has been calculated as

$$
\mathrm{S}_{\gamma}=\frac{\mathrm{N}_{\mathrm{o}} \cdot \mathrm{p} \cdot \mathrm{n}_{i} \cdot \mathrm{W} \cdot \varepsilon \cdot \tau}{\mathrm{A}_{\mathrm{T}}}\left[\left(1-\mathrm{e}^{-\mathrm{t}_{1} / \tau}\right) \cdot \mathrm{e}^{-\mathrm{t}_{2} / \tau} \cdot\left(1-\mathrm{e}^{-\mathrm{t}_{3} / \tau}\right)\right] \int_{\mathrm{o}}^{\infty} \phi(\mathrm{E}) \sigma(\mathrm{E}) \mathrm{dE},
$$

where $\mathrm{N}_{\mathrm{o}}$ is Avogadro's constant; $\mathrm{A}_{\mathrm{T}}$ is the atomic weight of the sample; $p$ is the probability of the excited nucleus decay by emitting the photon of energy $\mathrm{E}_{\gamma} ; \mathrm{n}_{i}$ is the relative fraction of the $i$-th isotope in the irradiated sample; $\mathrm{W}$ is the weight of the sample; $\varepsilon$ is the detector efficiency; $\tau$ is the average lifetime of the produced isotope $(\tau=1.442 \cdot \mathrm{T}$, where $\mathrm{T}$ is the half-life of the isotope); $t_{1}$ is the duration of the sample irradiation; $t_{2}$ is the time interval between the end of the irradiation and the start of the measurements; $t_{3}$ is the duration of the measurements; $\phi(\mathrm{E})$ is the differential flux of neutrons; $\sigma(\mathrm{E})$ and $\mathrm{E}$ are the reaction cross section and neutron energy, respectively. To fit the experimental data, $\phi(\mathrm{E})$ was chosen as a polynomial form multiplied by $\exp (-\alpha \mathrm{E})$ with the degree of the polynomial and exponential factor $\alpha$ taken as free parameters. Based on the experimental values of $\int_{0}^{\infty} \phi(\mathrm{E}) \sigma(\mathrm{E}) \mathrm{dE}$ and known dependences for $\sigma(\mathrm{E})[6]$, the shape of spectra $\phi(\mathrm{E})$ was reconstructed and the flux of fast neutrons was calculated. The accuracy of the values has achieved the level of $(10 \div 15) \%$.

The kinetic energy spectra of fast neutrons are shown in Fig. 2 for two irradiation setups. The average kinetic energy of the neutrons for the lead filter was found to be 1.5 $\mathrm{MeV}$ and the neutron flux in the energy interval of $(0.4-11) \mathrm{MeV}$ was $9.5 \cdot 10^{9} \mathrm{n} \mathrm{cm}^{-2} \mathrm{~s}^{-1}$. For the $(n / \gamma)$ converter, the flux was measured to be $4.0 \cdot 10^{8} \mathrm{n} \mathrm{cm}^{-2} \mathrm{~s}^{-1}$ and the average energy value was equal to $2.1 \mathrm{MeV}$.

Due to the high energy thresholds of the dosimeters, the measurement of the spectrum below $0.4 \mathrm{MeV}$ was not performed. It has been found via Monte-Carlo calculations [7] that the resonance neutron spectrum in the energy interval of $(0.1-0.4) \mathrm{MeV}$ is proportional to $\sim 1 /$ E. The total flux of these neutrons has been estimated by comparison with the experimental data for the fast neutrons at $\mathrm{E} \sim 0.4 \mathrm{MeV}$. It has been found that after the lead filter the neutrons of the $(0.1-0.4) \mathrm{MeV}$ energy range contributed to the fast neutron flux value of $\phi_{M C} \approx 30 \%$. 
The irradiated sample itself, as well as the surrounding equipment, may influence the measured spectra and the neutron flux value. So, the neutron fluence $\phi_{\text {meas }}(0.4-$ $11 \mathrm{MeV}$ ) after the lead filter was measured to be $9.5 \cdot 10^{9} \mathrm{n} \mathrm{cm}^{-2} \mathrm{~s}^{-1}$ in front of the cryostat and $4.8 \cdot 10^{9} \mathrm{n} \mathrm{cm}^{-2} \mathrm{~s}^{-1}$ behind it. The nickel foil as the integral monitor of the reactor power is mounted near the samples during the whole irradiation period. Also preliminary monitoring of the neutron flux and energy spectra is always necessary before a new irradiation run in order to fix the actual scenario of the exposure.

To compare the results of different radiation hardness tests, the fluence values have been expressed in effective values for neutrons with an equivalent energy of $1 \mathrm{MeV}$. The corresponding fluence $\phi_{\text {eff }}(1 \mathrm{MeV})$ is defined as

$$
\phi_{\text {eff }}(1 \mathrm{MeV})=\int_{E_{\min }}^{E_{\max }} \phi(E) \frac{k(E)}{k(1 \mathrm{MeV})} d E,
$$

where $\phi(\mathrm{E})$ is the measured neutron fluence and $k(\mathrm{E})$ is the energy dependent neutron kerma-factor ${ }^{1}$ for atomic displacements in GaAs (for the irradiation of the GaAs preamplifiers). Using the kerma-factor values for GaAs [8], the effective neutron fluence after the lead filter inside the cryostat with liquid nitrogen has been obtained:

$$
\begin{gathered}
\phi_{\text {eff }}(1 \mathrm{MeV}(\mathrm{GaAs}))=1.15 \cdot \phi_{\text {meas }}(0.4-11.0 \mathrm{MeV})+0.37 \cdot \phi_{M C}(0.1-0.4 \mathrm{MeV}) \\
=0.91 \cdot \phi_{\text {meas }}(0.1-11.0 \mathrm{MeV})=8.9 \cdot 10^{9} \mathrm{n} \mathrm{cm}^{-2} \mathrm{~s}^{-1} .
\end{gathered}
$$

\section{$4 \gamma$ Dosimetry}

Precise measurements of the $\gamma$ dose in the range of $\left(10^{5} \div 10^{6}\right)$ Gy, the area of interest for the radiation hardness tests, are not easy to accomplish at the reactor. The reason is the lack of $\gamma$ dosimeters which are completely insensitive to the neutron irradiation. In our experiment, thermoluminescent dosimeters TLD-700 [9] have been used to measure the doses from the $\gamma$ component of the mixed irradiation at the IBR-2 reactor. To measure the total dose values, alanine (PAD) and radiophotoluminescent dosimeters (RPL) with an upper limit of 1 MGy [10] have been employed.

The TLD detectors are based on LiF and have been produced as glass pills of $3.2 \times$ $3.2 \times 0.9 \mathrm{~mm}^{3}$ volume and weight of 24 milligrams. To decrease their sensitivity to low energy neutrons, the TLD-700 is enriched with the ${ }^{7} \mathrm{Li}$ isotope $\left(99.99 \%{ }^{7} \mathrm{Li}, 0.01 \%{ }^{6} \mathrm{Li}\right)$.

A special procedure was used to prepare TLD-700 for the irradiation. First, the TLD700 pills were annealed for 1 hour at a stable temperature of $(400 \pm 4)^{\circ} \mathrm{C}$ inside a titanium container [11]. After annealing, the container was positioned into the aluminium radiator to cool it to the optimal temperature in 15 minutes. Then the annealing procedure at $(100 \pm 2)^{\circ} \mathrm{C}$ was applied for 2 hours followed by the cooling. The standard calibration of

\footnotetext{
${ }^{1}$ kerma: kinetic energy released in matter
} 
the dosimeters has been performed with $10^{-2}$ Gy dose from a ${ }^{60}$ Co source. The thermoluminescent signals of the irradiated dosimeters were measured by means of a Harshaw model $2000(\mathrm{~A}+\mathrm{B})$ device.

The effect of "superlinearity" which is the nonlinear increase of signals for the doses higher than $1 \mathrm{~Gy}$, is known for TLD dosimeters [12, 13]. This threshold dose value is varied for different procedures to prepare the pills and "superlinearity" becomes more significant with the increase of the incoming photon energy.

The dependence of the thermoluminescent signal intensity of TLD-700 on the dose value was investigated using the monoenergetic $\gamma$ irradiation from ${ }^{137} \mathrm{Cs}\left(\mathrm{E}_{\gamma}=0.66 \mathrm{MeV}\right)$ and ${ }^{60} \mathrm{Co}\left(\mathrm{E}_{\gamma_{1}}=1.17 \mathrm{MeV}, \mathrm{E}_{\gamma_{2}}=1.33 \mathrm{MeV}\right)$ sources in the dose range from 1 Gy to $5 \cdot 10^{3}$ Gy. The results are presented in Fig. 3. The data obtained at the irradiation facility of the IBR-2 reactor with a broad energy spectrum of photons are shown as well. The detailed measurements of the $\gamma$ energy spectra at the IBR-2 reactor have not been performed yet, but some conclusions could be drawn based on the experimental data from the IBR-30 reactor [14]. In addition to the prompt irradiation from the reactor bursts, the neutron captures in the reactor shields provide a significant contribution to the spectrum. The $\gamma$ energy spectrum covers the energy range up to $10 \mathrm{MeV}$ with the average value of about $(1.5 \div 2 \mathrm{MeV})$.

To obtain the data, several TLD-700 dosimeters were positioned consecutively at the same locations and the dose value was determined by the time of exposure. The absolute values of the dose rates were calculated using the indication of TLD-700 at the $\gamma$ doses less than 1 Gy (linearity region). Absolute values of the $\gamma$ dose in the interval $(20-200)$ Gy were measured by means of the standard Fricke ferrosulphate dosimeters [15]. The results obtained from Fricke and TLD-700 dosimeters were in agreement within $( \pm 5 \%)$ for all the irradiation facilities used so far.

It is seen from Fig. 3 that "superlinearity" of TLD-700 dosimeters irradiated by "soft" photons from ${ }^{137} \mathrm{Cs}$ source becomes visible for the doses over 20 Gy and the maximal deviation (by factor of 3) from the linear behaviour is found at (400 - 600) Gy. The experimental data for ${ }^{60} \mathrm{Co}$ source and reactor irradiations are similar: "superlinearity" is evident beyond 10 Gy with the maximal deviation by factor of 4 at (300 - 1000) Gy. The curve shown in Fig. 3 for TLD-700 dosimeters was used as the calibration curve for the $\gamma$ dose monitoring at the IBR-2 reactor.

The PAD and RPL dosimeters have been used to measure the total integral dose at the IBR-2 facility for some of the irradiation test experiments.

An alanine dosimeter $[10,16,17]$ uses the stable paramagnetic centers generated by ionizing radiation in the $\alpha$-amino acid: $\mathrm{CH}_{3} \mathrm{CHNH}_{2}-\mathrm{COOH}$. An unpaired electron resulting from the breaking of a carbon covalent band is detected by the electron spin resonance (ESR) technique. Dosimeters of this type used in our work (4.8 mm diameter, $30 \mathrm{~mm}$ length, trade name: Elcugray), were based on a homogeneous mixture of polymer and alanine (33\%- $67 \%$ by weight).

Radiophotoluminescent dosimetry $[10,18]$ is based on the formation of stable colour 
centers in irradiated silver-activated aluminophosphate glass. The dosimeters used in this work ( $6 \mathrm{~mm}$ long and $1 \mathrm{~mm}$ in diameter) were of type DOS-2, produced by Schott, and had the following mixture (by weight): $53.7 \% \mathrm{O}, 33.4 \% \mathrm{P}, 4.6 \% \mathrm{Al}, 3.7 \% \mathrm{Ag}, 3.7 \% \mathrm{Li}$, $0.9 \%$ B.

The measurements of the irradiated PAD and RPL dosimeters have been undertaken at High Level Dosimetry (HLD) Laboratory at CERN by means of VARIAN-E3 ESR spectrometer (for PAD) and Toshiba FGD-6 reader (for RPL) [10]. The results are presented in Table 1 for the dose rates measured in the experiment on radiation hardness tests of GaAs electronics at the positions in front and behind the cryostat. The latter could be considered as an estimate of the dose level available at the area of the special container (see Fig. 1) with the samples to be irradiated in the simultaneous experiment (at optimal temperature) completing the irradiation of the large-scale samples.

The spatial distribution of the dose rate over the outer surface of the cylinder cryostat was measured by means of TLD-700 dosimeters. The $\gamma$ dose after the lead filter was found to be homogeneous within $( \pm 15 \%)$ over the whole irradiated volume and the averaged results from TLD-700 measurements, $\mathrm{D}_{\gamma}^{T L D}$, are presented in Table 1.

The total dose value, $\mathrm{D}_{\text {total }}^{P A D}$, was measured by means of alanine dosimeters. The contributions to this value from the neutrons of different energy ranges, $D_{1}$ and $D_{2}$, were also calculated:

$$
\mathrm{D}_{1}=\int_{0.4 \mathrm{MeV}}^{11 \mathrm{MeV}} k(E) \phi(E) d E, \mathrm{D}_{2}=\int_{0.03 \mathrm{MeV}}^{0.4 \mathrm{MeV}} k(E) \phi(E) d E
$$

where $\phi(E)$ is the measured differential neutron flux and $k(E)$ is kerma-factor for alanine [8].

For the lead filter, the neutrons were observed to contribute more than $(96 \%)$ of the total dose $\mathrm{D}_{\text {total }}^{P A D}$. The dose is dominated by fast neutrons with energy beyond $0.4 \mathrm{MeV}$. The contribution from neutrons of $(0.03-0.4) \mathrm{MeV}$ was estimated to be about $20 \%$ and it is negligible for lower energies.

For the $(n / \gamma)$ converter, the main contribution to the total dose $\mathrm{D}_{\text {total }}^{P A D}$ is provided by photons (85\%). The homogeneity of the radiation field was checked by means of TLD700 dosimeters. The $\gamma$ field is homogeneous in the region around the cryostat in the vertical direction within $\pm 6 \%$, but the converter causes the gradient of the $\gamma$ dose when the distance grows from its surface.

In general, a satisfactory agreement between the $\gamma$ doses measured by means of TLD700 and alanine dosimeters for both experimental setups was found after substraction of the neutron contribution from the $\mathrm{D}_{\text {total }}^{P A D}$ value.

Under conditions of the intensive neutron field which prevails in the setup with the lead beam filter, the thermal neutrons provide the major contribution to the dose measured by means of RPL dosimeters via the neutron capture reactions in the detector materials. These reactions $\left({ }^{6} \mathrm{Li}(\mathrm{n}, \alpha){ }^{3} \mathrm{~T},{ }^{10} \mathrm{~B}(\mathrm{n}, \alpha){ }^{7} \mathrm{Li}, \mathrm{Ag}(\mathrm{n}, \gamma) \mathrm{Ag}\right)$ have large cross sections to absorb slow neutrons. The detailed analysis of the RPL properties is beyond the scope of the present article. Nevertheless, it should be emphasized that for the $(n / \gamma)$ converter 
when the initial neutron field is suppressed significantly, the dose measured by means of RPL dosimeters agrees with those obtained by TLD-700 and PAD dosimeters.

\section{Conclusions}

The facility aimed at studying radiation hardness properties of various materials of large dimensions (up to an area of $800 \mathrm{~cm}^{2}$ ) in a wide range of temperatures, is described in the article. The radiation hardness tests of GaAs preamplifiers and different materials for the ATLAS detector at LHC have been performed successfully at this facility.

Two configurations of radiation field were used for irradiation. For the neutron irradiation, the primary $\gamma$ radiation is suppressed by the lead absorber and the radiation field is composed by fast neutrons with the flux value of $1.4 \cdot 10^{10} \mathrm{n} \mathrm{cm}^{-2} \mathrm{~s}^{-1}$ in the energy range $(0.1-11) \mathrm{MeV}$. The remaining $\gamma$ dose rate is decreased to the level of $50 \mathrm{~Gy} / \mathrm{h}$ which is less than $4 \%$ of the total radiation dose.

For $\gamma$ irradiation, the flux of fast neutrons is attenuated by the $(n / \gamma)$ converter and the radiation field is composed by the primary $\gamma$ irradiation from the reactor and by the photons produced via the neutron capture in the cadmium material of the converter. The $\gamma$ dose rate is about $500 \mathrm{~Gy} \mathrm{~h}^{-1}$ (about $85 \%$ of the total dose). The fast neutron flux value for this configuration of the radiation field is $4 \cdot 10^{8} \mathrm{n} \mathrm{cm}^{-2} \mathrm{~s}^{-1}$.

For both configurations of the radiation field, thermal neutrons were suppressed by introducing the boron carbide filter.

\section{Acknowledgements}

The authors acknowledge the Department of Radiation and Radiobiological Research of JINR for the opportunity to work with TLD-700 dosimeters. C.L. and E.L-F. thank NSERC of Canada for their support. We would like to thank M.Tavlet from CERN-TIS Division for providing the alanine and RPL dosimeters and their data processing.

\section{References}

[1] ATLAS Collaboration, Technical Proposal, CERN/LHCC/94-43 (1994).

[2] RD33 Collaboration, C. Berger et al., Nucl.Instr.Methods, A357 (1995) 333.

[3] A. Cheplakov et al., JINR Preprint, E13-96-358, Dubna (1996) (submitted to NIM).

[4] E. Shabalin, Fast pulsed and burst reactors, Pergamon Press Ltd., Oxford (1979).

[5] ATLAS Collaboration, Technical Design report, CERN/LHCC/96-41 (1996). 
[6] V. McLane, C.L. Dunford and. P.F. Rose, Neutron Cross Section, Vol.2, Academic Press Inc., (1988).

[7] V.F. Peresedov, A.D. Rogov, JINR Rapid Communication, 1-[75]-96, Dubna (1996) 69.

[8] A.M. Ougouag et al., IEEE Trans. Nucl. Sci. NS-37 (1990) 2219.

[9] Harshaw Chemical Co., Cleveland, Ohio 44106, USA.

[10] E. León-Florián, H. Schönbacher and M. Tavlet, CERN/TIS-CFM/IR/93-03 (1993).

[11] G.Ya. Kaskanov and M.M. Komochkov, JINR Communication, 16-83-452, Dubna (1983).

[12] K.K. Schwarts, Z.A. Grant et al., Thermo-luminescent dosimetry (in Russian), Zinatne, Riga (1968).

[13] F.M. Gayton, J.R. Harvey and J.H. Jackson, J. Brit.Nucl. Energy, 11 (1972) 125.

[14] V.A. Khitrov and Pack Hong-Cher, JINR Internal Report, Dubna (1974).

[15] K.K. Aglintsev, Ionising irradiation dosimetry (in Russian), Gostekhizdat, Moscow (1957).

[16] F. Coninckx, H. Schönbacher, Appl. Radiation and Isotopes, 44 (1993) 67.

[17] F. Coninckx, H. Schönbacher et al., Appl. Radiation and Isotopes, 40 (1989) 977.

[18] C. Coche et al., CERN TIS Commission Report, TIS-RP/205 (1988). 


\section{TABLE CAPTIONS}

Table 1: Dose rate values (in Gy/h) for different beam filters in front and behind the cryostat (see Fig.1). 


\section{FIGURE CAPTIONS}

Fig. 1: Schematic layout of the experimental set-up: (1) motherboard with the electronics; (2) cryostat; (3) beam filter; (4) boron carbide layer; (5) support frame; (6) movable shielding platform; (7) reactor beam shutter; (8) inner and (9) outer reactor shields; (10) cryogenic lines; (11) signal cables; (12) transporting tube for the samples; (13) neutron and $\gamma$ dosimeters; (14) nickel foil.

Fig. 2: The kinetic energy spectra of the neutrons from the IBR-2 reactor.

Fig. 3: Dependence of the thermo-luminescent signal intensity of TLD-700 on the dose value for the irradiation from ${ }^{137} \mathrm{Cs}$ and ${ }^{60} \mathrm{Co} \gamma$ sources and at IBR-2 reactor. 
Table 1

\begin{tabular}{|c|c|c|c|c|}
\hline Doses & \multicolumn{2}{|c|}{ after lead filter } & \multicolumn{2}{c|}{ after $(n / \gamma)$ converter } \\
\hline measured & in front & behind & in front & behind \\
\hline $\mathrm{D}_{\gamma}^{T L D}$ & \multicolumn{2}{|c|}{$48 \pm 8$} & $(466 \pm 40)$ & $(170 \pm 10)$ \\
$\mathrm{D}_{\text {total }}^{P A D}$ & $1164 \pm 16$ & $435 \pm 2$ & $627 \pm 19$ & $216 \pm 4$ \\
$\mathrm{D}_{1}$ & $886 \pm 90$ & $387 \pm 40$ & $58 \pm 10$ & $25 \pm 4$ \\
$\mathrm{D}_{2}$ & $240 \pm 20$ & $31 \pm 5$ & $10 \pm 2$ & $4 \pm 1$ \\
$\mathrm{D}_{\gamma}^{P A D}$ & \multicolumn{2}{|c|}{$25 \pm 37$} & $(559 \pm 22)$ & $(187 \pm 9)$ \\
$\mathrm{D}_{\text {total }}^{\text {RPL }}$ & $759 \pm 60$ & $195 \pm 27$ & $673 \pm 89$ & $226 \pm 51$ \\
\hline
\end{tabular}




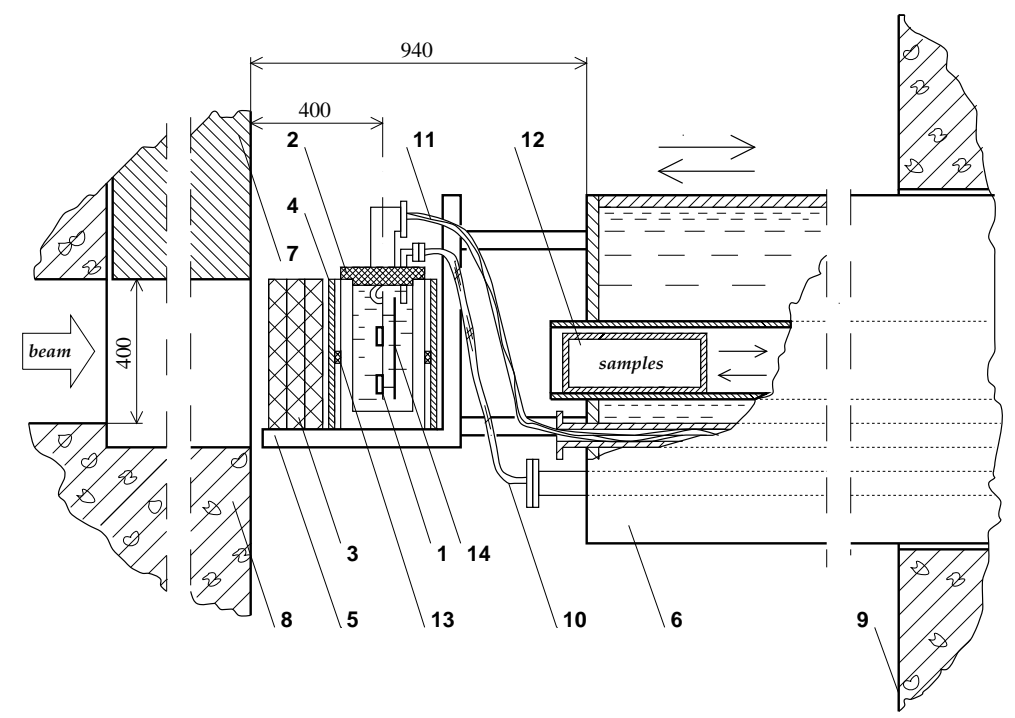

Figure 1: 


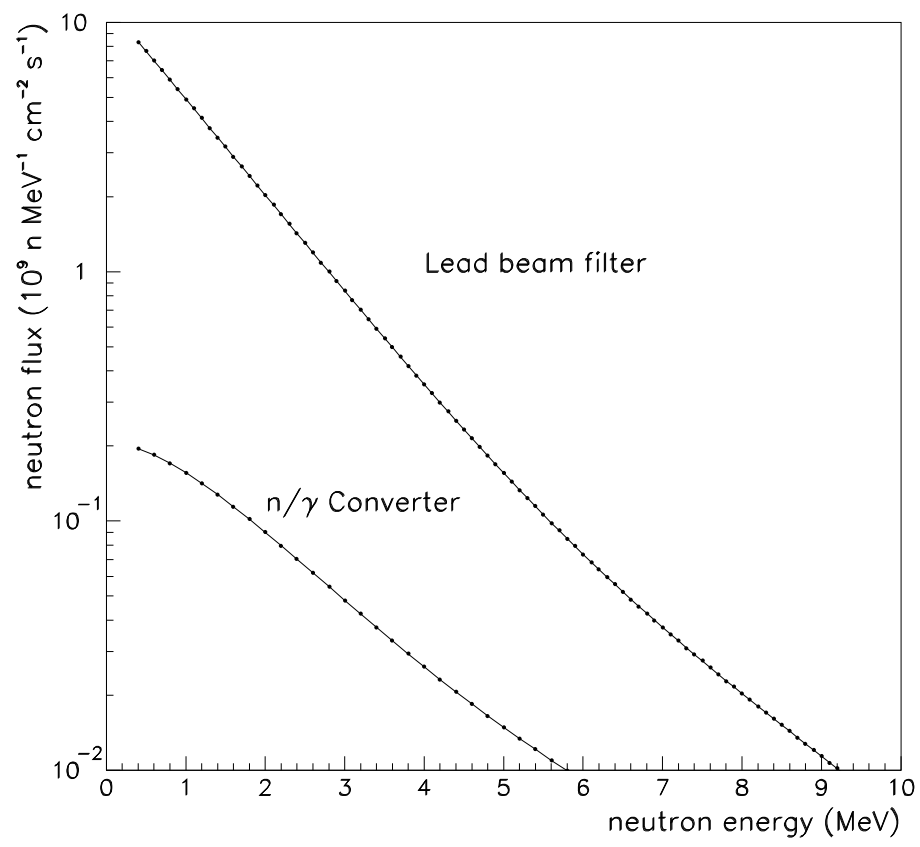

Figure 2: 


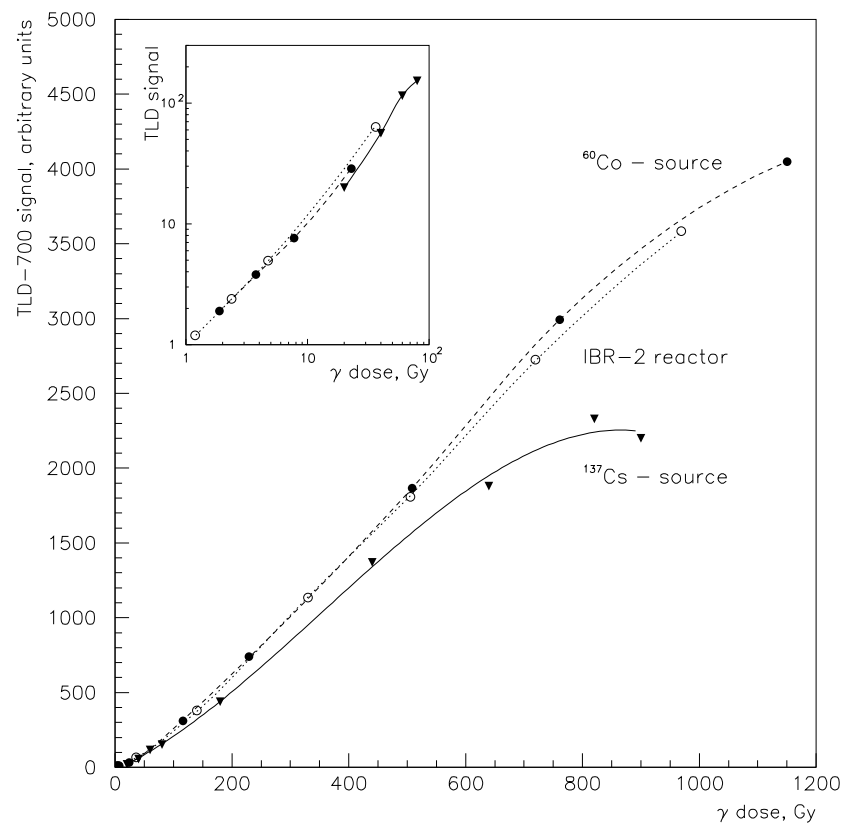

Figure 3: 